\title{
Synthetic Stellar libraries and SSP simulations in the Gaia Era
}

Antonella Vallenari ${ }^{1}$, Rosanna Sordo ${ }^{1}$, Rosaria Tantalo ${ }^{2}$, France Allard $^{3}$, Ronny Blomme ${ }^{4}$, Jean-Claud Bouret ${ }^{5}$, Ines Brott ${ }^{6}$, Yves Fremat $^{4}$, Christophe Martayan ${ }^{4}$, Yassine Damerdji ${ }^{7}$, Bengt Edvardsson ${ }^{8}$, Eric Josselin ${ }^{9}$, Bertrand Plez ${ }^{9}$, Oleg Kochukhov ${ }^{10}$, Mary Kontizas $^{11}$, Andreas Schweitzer ${ }^{12}$, Jean Zorec ${ }^{4}$, Paraskevi Tsalmantza ${ }^{13}$, Ulisse Munari ${ }^{1}$ \& Tenay Saguner ${ }^{1,2}$

${ }^{1}$ INAF - Osservatorio Astronomico di Padova ${ }^{2}$ Astronomy Department, Padova University

${ }^{3}$ CRAL-ENS Lyon (France) ${ }^{4}$ Royal Observatory, Brussels (Belgium) ${ }^{5}$ CNRS Marseille (France) ${ }^{6}$ Sterrenkundig Instituut Utrecht (The Netherlands) ${ }^{7}$ Univ. De Liege (Belgium) ${ }^{8}$ Mid Sweden University Sundsvall (Sweden) ${ }^{9}$ GRAAL Montpellier (France) ${ }^{10}$ Uppsala University (Sweden) ${ }^{11}$ Athens University (Greece) ${ }^{12}$ Hamburger Sternwarte, Hamburg, (Germany) ${ }^{13}$ MPI fr Astronomie, Heidelberg, (Germany)

\begin{abstract}
The Gaia mission will obtain accurate positions, parallaxes and proper motions for $10^{9}$ object all over the sky. In addition, it will collect low resolution spectroscopy in the optical range for $\sim 10^{9}$ objects, stars, galaxies, and QSOs. Parameters of those objects are expected to be part of the final Catalog. Complete and up-to-date libraries of synthetic stellar spectra are needed to train the algorithms to classify this huge amount of data. Here we focus on the use of the synthetic libraries of spectra calculated by the Gaia community to derive grids of Single Stellar Populations as building blocks of population synthesis models.
\end{abstract}

Keywords. Gaia; stars: synthetic spectra; population synthesis

\section{Gaia Stellar and Single Stellar Population libraries}

Large and up-to-date libraries of spectra are of fundamental importance for the Gaia mission (to be launched early 2012). They are used as templates to train supervised algorithms for an automatic classification of the Gaia spectra (Bailer-Jones et al. 2008). State-of-the-art synthetic stellar libraries are calculated by the Gaia community with an homogeneous coverage of parameter space at $0.1 \mathrm{~nm}$ sampling in the optical range (300-1100 nm) for stars spanning the most different types, from M to O, from A-peculiar to Emission lines to White Dwarfs (Bouret et al. 2008, Shulyak et al. 2004, Gustafsson et al. 2008, Alvarez et al. 1998, Brott et al. 2005, Allard et al. 2000, Martayan et al. 2008, Hindson et al. 2008). More detail can be found in Sordo et al. (2009). In addition, we make use of the Munari et al. (2005) synthetic stellar library, which is based on Kurucz's codes and allows to investigate the effects of $[\alpha / \mathrm{Fe}]$ variations up to relatively high $\mathrm{T}_{\text {eff }}$. This library (in its solar-scaled version) was extended in the UV, by the UVBlue library (Rodríguez-Merino et al. 2005) which uses the Kurucz's model atmospheres and covers the 85-470 $\mathrm{nm}$ spectral range. Here we focus on the use of these spectra to built synthetic libraries of single stellar populations (SSPs) to be used in the population synthesis study of star clusters and galaxies (we quote among others Vallenari et al. 2008, Tsalmantza et al. 2007, 2009). SSPs are derived as described in Tantalo (2005). We calculate three sets at changing parameters: a) Optical-UV solar-scaled SSPS: using Padova isochrones by Bertelli et al. (1994) together with the Munari et al. (2005) library extended by UVBlue library. The SSPs have $[\alpha / \mathrm{Fe}]=+0.0 ; \mathrm{b})$ Optical solar-scaled SSPs: the Padova isochrones 
by Bertelli et al. (1994) are coupled with the Gaia libraries. The SSPs have $[\alpha / \mathrm{Fe}]=+0.0$; c) Optical $\alpha$-enhanced SSPs: Teramo isochrones by Pietrinferni et al. (2006) are coupled with the Munari et al. (2005) library. The SSPs have $[\alpha / \mathrm{Fe}]=+0.4$. Extensive tests on the results (Lick indexes calculation, comparison with spectra of observed clusters) will be presented in Vallenari et al. (2009) (in preparation). The comparison of Lick indexes calculated from our SSP library ( $\alpha$-enhanced version) with the observational data on Galactic and M31 clusters from Trager et al. (1998) shows a good agreement (see Fig. 1).

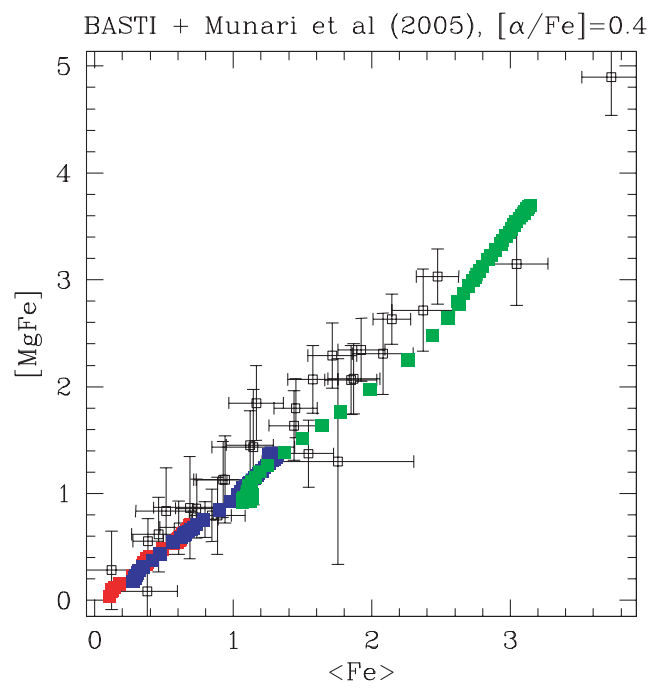

Figure 1. Lick indexes $\langle\mathrm{Fe}\rangle$ vs $\mathrm{MgFe}$ calculated using Munari et al. (2005) library, BASTI tracks, $[\mathrm{Fe} / \mathrm{H}]=-2.0$ (red), -1.5 (blue), -0.5 (green), $[\alpha / \mathrm{Fe}]=+0.4$ are compared with Trager et al. (1998) data on globular and M31 clusters (open squares)

\section{References}

Alvarez, R. \& Plez, B. 1998, AJ 330, 1109

Allard, F., Hauschildt, P. H., \& Schweitzer, A. 2000, ApJ, 539, 366

Bailer-Jones, C. A. L., Smith, K. W., Tiede, C., Sordo, R., \& Vallenari, A. 2008, MNRAS, 391, 1838

Bertelli, G., Bressan, A., Chiosi, C., Fagotto, F., \& Nasi, E. 1994, A\&SAS, 106, 275

Bouret, J.-C. et al. 2008, in preparation

Brott, I. \& Hauschildt, P. H. 2005, ESA SP-576, 565

Castanheira, B. G. et al. 2006, A\& A, 450, 331

Gustafsson, B., Edvardsson, B., Eriksson, K. et al. 2008, A\& A, 486, 951

Hindson, L., Napiwotzki, R., Heber, U., \& Lemke, M. 2008, ASP-CS 392, 163

Martayan, C. et al. 2008, SF2A-2008, 499

Munari, U., Sordo, R., Castelli, F., \& Zwitter, T. 2005, A\&A 442, 1127

Pietrinferni, A., Cassisi, S., Salaris, M., \& Castelli, F. 2006, ApJ, 642, 797

Rodríguez-Merino, L. H., Chavez, M., Bertone, E., \& Buzzoni, A. 2005, ApJ, 626, 411

Shulyak et al. 2004,AEA A, 428, 993

Sordo, R. et al. 2009, MemSAI, 80, 103

Tantalo, R. 2005, The Initial Mass Function 50 Years Later, 327, 235

Trager, S. C., Worthey, G., Faber, S. M., Burstein, D., \& Gonzalez, J. J. 1998 ApJS, 116, 1

Tsalmantza, P. et al. 2007, A\&A A, 470, 761

Tsalmantza, P. et al. 2009, arXiv:0907.1671

Vallenari, A. \& Sordo, R. 2008, arXiv:0812.0293 